\title{
Isolating phonological components that increase tip-of-the-tongue resolution
}

\author{
LISE ABRAMS, KATHERINE K. WHITE, and STACY L. EITEL \\ University of Florida, Gainesville, Florida
}

\begin{abstract}
Three experiments investigated the role of specific phonological components in priming tip-of-thetongue (TOT) resolution. When in a TOT state, participants read a list of words that included phonological primes intermixed among unrelated words. The phonological primes contained either the same first letter as the target (Experiment 1), a single syllable (first, middle, or last) of the target (Experiment 2), or the first phoneme or first syllable of the target (Experiment 3). Reading first-letter primes in Experiment 1 did not help to resolve TOTs, whereas reading first-syllable primes significantly increased word retrieval in Experiment 2. Experiment 3 replicated the results of Experiments 1 and 2 using firstphoneme primes instead of first-letter primes and using two primes instead of three, although firstsyllable priming occurred only for primes read silently. The results of these experiments support a transmission deficit model, where TOTs are caused by weak connections among phonological representations and can be resolved through internal or overt production of specific phonology.
\end{abstract}

A tip-of-the-tongue (TOT) state occurs when a word is temporarily inaccessible for retrieval, despite a feeling of knowing the word. Although the cause of TOT states has been debated (see A. S. Brown, 1991, for a review), one predominant view is that the target word does not receive sufficient activation to allow for word retrieval (e.g., R. Brown \& McNeill, 1966; Meyer \& Bock, 1992; Yaniv \& Meyer, 1987). A more specific account comes from the transmission deficit (TD) model of TOT states (Burke, MacKay, Worthley, \& Wade, 1991; James \& Burke, 2000; MacKay \& Burke, 1990; Rastle \& Burke, 1996), which states that TOTs are caused by weak connections between lexical nodes and phonological nodes that are represented in a hierarchical network of multilevel systems (see Burke et al., 1991; MacKay, 1987; MacKay \& Burke, 1990, for a description of this network called node structure theory). The present experiments tested this account of TOT states by strengthening connections to specific phonological nodes and assessing the effects on TOT resolution. These experiments investigated which phonological features were capable of

This research was partially supported by APA Division 20 and the Retirement Research Foundation through a master's research proposal award granted to K.K.W. Portions of this research were reported at the University of Florida Graduate Student Forum in April 1999, the annual meeting of the Psychonomic Society in November 1999, and the annual meeting of the American Psychological Society in May 2003. Portions of Experiment 3 were taken from S.T.E.'s senior thesis, which appears in the October 2003 issue of the University of Florida's Journal of Undergraduate Research. We thank Debby Burke, Lori James, and Don MacKay for their helpful comments on an earlier draft of this article, and we thank Arielle Borovsky, Melinda Cothern, Chris Hadley, Sean Hoover, and Paul McDermott for assistance in data collection. K.K.W. is now at Educational Testing Service, Princeton, New Jersey. Correspondence should be addressed to L. Abrams, Department of Psychology, University of Florida, P. O. Box 112250, Gainesville, FL 32611-2250(e-mail: abrams@ufl.edu). facilitating word retrieval by priming specific phonological features - the first letter of the target word, the first phoneme, or a single syllable-during TOT states.

Node structure theory and the TD model offer a specific explanation for why TOT states occur and how they can be resolved by postulating two processes that determine word retrieval: node priming and node activation. Node priming is a form of excitation that is transmitted across connections between nodes, preparing them for activation (other network models refer to this as "spreading activation," e.g., Anderson, 1983; Collins \& Loftus, 1975). Node activation is an "all-or-none" process that occurs when the level of node priming exceeds the threshold that is required for word retrieval. According to the TD model, a TOT state occurs when the connections to a word's phonological nodes are weakened, causing a reduction in the transmission of node priming to these nodes. Without a sufficient amount of node priming, these phonological nodes cannot get activated, and therefore word retrieval cannot occur. Connections to a word's phonological nodes weaken over time if they have not been activated frequently or recently. For example, if the lexical node for the word contraband has not been activated often in the past several years, a TOT is more likely to occur during word retrieval because the connections to its phonological nodes (the sounds /kŏn/, /trə/, and /bănd/) are weakened.

However, some aspects of phonology, such as the first letter or phoneme, can be retrieved during a TOT state in spite of the inability to retrieve the entire word (Burke et al., 1991; Harley \& Bown, 1998; Meyer \& Bock, 1992; Miozzo \& Caramazza, 1997). Within the TD model, this "partial retrieval" occurs because sufficient node priming accumulates on a particular phonological node and leads to activation of the corresponding phonological component. For example, the first phoneme of the word contra- 
band may get activated and result in the feeling that the word begins with $c$. However, for complete word retrieval to occur, every phonological node must be activated.

Previous researchers have attempted to activate the missing phonology and increase TOT resolution by implementing an intentional cuing paradigm (e.g., Bowles \& Poon, 1985; Jones, 1989; Jones \& Langford, 1987; Maylor, 1990; Meyer \& Bock, 1992; Perfect \& Hanley, 1992). For example, Meyer and Bock presented participants having a TOT state with a word that shared multiple phonological features with the TOT target word (e.g., the initial sound and letter, number of syllables, and stress pattern) and informed them that the cue "may help you to think of the word" (p. 719). They found that more TOT states were resolved when participants were presented with phonological cues than semantic or unrelated cues, suggesting that phonological cues facilitate retrieval during TOT states, as predicted by the TD model (see Jones, 1989, and Jones \& Langford, 1987, for exceptions). However, facilitation from cues in their study most likely reflected strategic retrieval processes. Knowing that the cue may be related to the target word may encourage a directed memory search for the target, using phonological properties of the cue. Although cuing produces facilitation in word retrieval, the nature of this facilitation is different from the facilitation produced by automatic retrieval processes that occur during a TOT state.

To minimize the influence of conscious retrieval strategies on word retrieval during a TOT state, James and Burke (2000, Experiment 2) employed a type of modified repetition priming paradigm that disguised the relation between the primes and the TOT target word. After indicating that they were in a TOT state, participants pronounced aloud and rated the pronunciation difficulty of 10 words, 5 of which cumulatively contained all of the target word's phonology. The results showed that participants resolved more TOT states following phonological primes than unrelated words. Even though these results are consistent with cuing paradigms, they are more likely to accurately reflect the automatic processes involved in normal word retrieval (i.e., words "popping" into mind instead of being accessed through conscious search strategies). In terms of node structure theory and the TD model, James and Burke concluded that a relatively long-term change in connection strength (which occurs without an individual's awareness) is responsible for increased target word retrieval in the phonological priming paradigm.

Like James and Burke (2000), we assessed TOT resolution following presentation of phonological primes, not cues. However, our experiments primed participants with words that contained specific aspects of phonology (e.g., first letter/phoneme or a single syllable), unlike James and Burke and cuing studies that have used words that shared several phonological characteristics with the target word. The present experiments are the first to explore the effect of isolated phonological features on TOT resolution, independent of conscious retrieval strategies.

Node structure theory and the TD model can be used to predict the aspects of phonology that are most likely to facilitate TOT resolution. Specifically, they predict that the critical phonological aspects necessary for word retrieval are likely to be located in the initial portion of the word. In node structure theory, language production involves sequential left-to-right activation (MacKay, 1987): One must activate the phonological nodes at the beginning of a word before activating the nodes in the next segment, and so forth, until the entire word is activated and can be produced. If the initial segment fails to get activated, then subsequent portions of a word cannot get activated, which results in a TOT state. Furthermore, the sequential nature of activation suggests that the initial portion of the word is also critical for cuing or priming word retrieval, in such a way that strengthening connections to a word's initial phonological nodes is necessary for activation of the remaining segments during a TOT state. This prediction has been supported by research on cuing with different parts of target words, which has shown that the initial part of a word (e.g., first letter or sound) serves as the best retrieval cue (e.g., Bowles \& Poon, 1985; Freedman \& Landauer, 1966; Gruneberg \& Monks, 1974; Heine, Ober, \& Shenaut, 1999), especially in comparison to middle or end letters (Horowitz, White, \& Atwood, 1968).

In contrast to activation of the initial segment, failed activation of a word's middle or final segments is less likely to have an effect, either in causing TOT states or in facilitating TOT resolution. Even if the latter segments do not get activated immediately, the initial phonology that is available can be used to retrieve the remaining phonology and enable word retrieval, preventing a TOT state from occurring. In terms of TOT resolution, activation of the middle or final segments during a TOT state does not ensure that the initial segment will get activated, resulting in no increase in word retrieval following cues or primes containing the latter syllables. Latter syllables are also likely to be less effective primes than initial syllables because of inherent characteristics of syllables at the end of words. Certain parts of speech often have similar endings (e.g., many nouns end in the suffix tion, and many verbs end in the suffix ate), which make them more predictable. Previous research supports this claim by demonstrating a greaterthan-chance probability of guessing the final letters of words during a TOT state (e.g., Kohn et al., 1987; Koriat \& Lieblich, 1975; Rubin, 1975). Because many words share these endings, the latter syllables will be used more often, and their higher frequency of use will make the connections to their phonological nodes stronger and less susceptible to deficits in the transmission of node priming across these connections. However, unless the first and middle syllables are activated, the last syllable will not be retrieved. Consistent with these predictions and the partial information that people often report when in a TOT state, we designed our studies to test the effects of first letter (Experiment 1), a specific syllable (Experiment 2), and first phoneme versus first syllable (Experiment 3 ) on TOT resolution.

\section{EXPERIMENT 1}

In this experiment, we investigated whether primes with the same first letter as the target (and same first phoneme 
$82 \%$ of the time) facilitate TOT resolution. As discussed earlier, node structure theory postulates that activation is a sequential process; therefore, the initial sound of a word must be activated before later sounds can be activated. Consequently, providing the first letter should activate the initial sound, boost the transmission of priming to the other phonological nodes, and facilitate TOT resolution.

\section{Method}

Participants. Sixty undergraduates (14 male, 46 female; mean age 18.9 years, $S D=1.5$ ) were recruited from introductory psychology classes and cognitive psychology classes at the University of Florida. Participants received either partial course credit or extra credit for their participation. All participants reported normal or corrected-to-normal vision and were native English speakers.

Materials. Seventy-eight target words and their corresponding general knowledge questions for inducing TOT states were taken from previous studies and word norms (Burke et al., 1991; Francis \& Kučera, 1982; Jones, 1989; Kohn et al., 1987; Meyer \& Bock, 1992; Nelson \& Narens, 1980). The answers to these questions (i.e., target words) were mainly low frequency $(0-10$ per million, with the exception of three words, one at 13 per million and two at 21 per million), did not include proper names, and were three or four syllables long.

For each target word, two lists were generated. The unrelated list contained seven filler words that were phonologically and semantically different from the target. The other list was a "primed" list that contained four of the filler words from the unrelated list along with three primes that shared the first letter with the target. Across all lists, half of the primed lists shared the same number of syllables with the target, whereas the other half contained a different number of syllables. Appendix A illustrates an example of the primed and unrelated lists. Filler words ranged from one to five syllables, were never the same number of syllables as the target word for that list, and never shared the first letter with the target. The primes were positioned in the list so that no two primes appeared consecutively; half of the word lists included primes at Positions 1, 3, and 6 in the list, and the other half of the word lists had primes in Positions 2, 4, and 7. Following TOT and "don't know" responses, the computer program randomly chose one of the two lists to be presented for a given target word. Following "know" responses, five words from the unrelated list were presented. Although unnecessary because the question was answered, presenting words after a "know" response allowed words to be presented after every question, regardless of response, which was intended to minimize participants' likelihood of seeing a relation between the primes and targets.

After all 78 general knowledge questions had been presented, a multiple-choice recognition test was given that presented all of the unresolved questions along with four possible answers: the correct (target) answer, a word that was semantically related to the target, a word that was phonologically related to the target, and a word that was semantically and phonologically unrelated to the target. The purpose of the recognition test was to verify that participants' TOT responses were for the intended target words. Targets that were not selected during the recognition test were considered incorrect TOT responses and were excluded from all analyses.

A postexperiment questionnaire was administered verbally to assess participants' awareness of the priming manipulation and to evaluate participants' intent to use the primes as cues to facilitate retrieval. The questionnaire consisted of a series of increasingly specific questions that determined whether participants were aware of any relation between the reading words task and the general knowledge questions (i.e., awareness), and whether they intentionally used the reading words to try to generate answers to the general knowledge questions (i.e., intent).

The experiment was performed on Pentium II 350-MHz IBMcompatible computers using a computer program written in Visual Basic 5.0. The screen color was a light blue, and all text was printed in black, boldface, lowercase Times New Roman. The general knowledge questions were presented in a 24-point font size, and the primes were presented in a 36-point font size. Both the general knowledge questions and the primes were centered on the screen.

Design and Procedure. The experiment featured a single-factor design, with priming condition (first-letter primes vs. unrelated fillers) as a within-participants factor. Prior to the experiment, participants were given an explanation of the TOT phenomenon as a word that one is certain that he/she knows but cannot retrieve at the moment. A general knowledge question was presented in the center of the screen, and participants were asked to state whether they knew the answer, did not know, or were in a TOT state. If they knew the answer, a subset of five words from the unrelated list appeared one word at a time. If they did not know the answer or were in a TOT state, a primed list or unrelated list was presented at a rate of $2.5 \mathrm{sec}$ per word. For all lists, participants read each word either aloud or silently, with type of production blocked for each half of the experiment. The general knowledge question was presented again for the participant's response. After all 78 questions had been presented, a four-choice recognition test was given for questions corresponding to "don't know" and TOT responses that had not been resolved, and then the postexperiment questionnaire was administered. All responses made by the participants were tape-recorded and doublechecked for accuracy when necessary.

\section{Results and Discussion}

Responses on the postexperiment questionnaire were analyzed to evaluate participants' awareness of the priming manipulation and intent to use the primes as cues to facilitate retrieval. The majority of participants reported having no awareness of a relation between the primes and the target word (65\%) or were "incorrectly" aware and described a nonexistent relation between the primes and targets (e.g., they were synonyms [24\%]). Only $11 \%$ of the participants were correctly aware of a phonological relation and described the relation between the primes and targets as sharing the same first letter or sound. Only 2 participants (4\%) stated that they were able to exercise intent (i.e., deliberately attempt to use the primes to facilitate retrieval). These 2 participants as well as an additional 4 participants who did not complete the postexperiment questionnaire were excluded from all analyses to ensure that conscious retrieval strategies were not used during the priming task. We used the criterion of intent rather than awareness as a basis for exclusion because prior research using traditional repetition priming paradigms has shown that intent (demonstrated via explicit retrieval strategies) is more critical than awareness in influencing priming, both for single words (e.g., Bowers \& Schacter, 1990) and for new associations (e.g., McKone \& Slee, 1997). Two additional participants were excluded because of computer error, yielding 52 remaining participants whose data were used in all analyses.

In terms of the initial responses to the general knowledge questions, $34 \%$ were "know" responses, $10 \%$ were TOT responses, and $32 \%$ were "don't know" responses. These percentages include only "correct" responses, where participants provided a correct answer to the question for a "know" response or a correct response on the recognition test for an unresolved TOT response. Incorrect "know" responses (17\%) and incorrect TOT responses $(7 \%)$ were excluded from all analyses. 
Table 1

Experiment 2: Word Retrieval (Percent) Following Primes and Unrelated Words After an Initial TOT Response

\begin{tabular}{lcc}
\hline Priming Condition & Mean Resolution & $S D$ \\
\hline Same first syllable & 50.4 & 37.7 \\
Same middle syllable & 32.9 & 34.2 \\
Same last syllable & 22.6 & 25.2 \\
Unrelated & 24.9 & 30.6 \\
\hline
\end{tabular}

A paired-samples $t$ test was performed on percentage of correct word retrieval following TOT responses. Excluding 2 participants (4\%) for not having at least one TOT response in each of the two conditions, the $t$ test revealed no significant difference in TOT resolution for first-letter primes $(M=44.7 \%, S D=36.4 \%)$ relative to the unrelated list $(M=40.4 \%, S D=34.4 \%)[t(49)=.79$, $S E=5.5, p>.433]$.

Experiment 1 demonstrated no priming effects on TOT resolution, contrary to the predictions of node structure theory and the TD model. When participants were in a TOT state, giving them primes that contained the first letter of the target did not increase the probability of TOT resolution. These results differ with cuing studies of TOT states that demonstrated increased word retrieval following presentation of the first letter of the target (e.g., Freedman \& Landauer, 1966; Heine et al., 1999) or both the first letter and number of syllables in the target (e.g., Bowles \& Poon, 1985; Maylor, 1990; Meyer \& Bock, 1992; Perfect \& Hanley, 1992). Therefore, facilitation from first-letter cues seen in previous studies, which did not make efforts to disguise the relation between the cues and targets, most likely reflects strategic retrieval processes such as guessing strategies, where conscious efforts to retrieve words increase word retrieval.

The lack of phonological priming indicates that a single letter was insufficient to overcome the transmission deficits that cause TOT states. Within the TD model, one explanation for this insufficiency is that activation of the first letter sends node priming to numerous words that contain that initial letter. As a result, the desired syllable may not be activated because many syllable nodes are receiving node priming from the first letter. This interpretation suggests that increasing the amount of phonological information during priming may lead to facilitation in TOT resolution.

\section{EXPERIMENT 2}

The purpose of this experiment was to increase the number of phonemes primed to a syllable unit and to compare the effectiveness of priming different syllable positions. Primes were constructed so that they contained one of three possible syllable positions (first, middle, or last) to determine what specific portions of a word are more susceptible to transmission deficits. On the basis of node structure theory's postulation of sequential activation and results of prior cuing studies, we predicted that first- syllable priming would be sufficient to activate the remaining phonology and allow for complete retrieval of that word. In contrast, middle- and last-syllable priming should have little effect on TOT resolution because the initial syllable must be activated first for successful word retrieval to occur. In addition, cuing studies have failed to establish a link between non-initial word phonology and word retrieval in episodic memory tasks (e.g., Horowitz et al., 1968).

\section{Method}

Participants. Sixty undergraduates (14 male, 46 female; mean age 20.3 years, $S D=1.6$ ) were recruited from the same sources as those in Experiment 1, although none of the participants had participated in Experiment 1.

Materials. Ninety general knowledge questions that had target answers three or four syllables in length were used in this experiment. Fifty-eight questions that tended to elicit high numbers of TOTs were taken from Experiment 1, and 32 new questions were added. Of these 90 questions, 15 targets were fillers because they were always primed for only one specific syllable across participants and were therefore excluded from analyses. The recognition test (except for those items that were added or deleted) and postexperiment questionnaire were identical to those used in Experiment 1.

For each target word, the number of filler words in each of the four lists was increased from Experiment 1 to reduce the likelihood that participants would deduce that the words they read while in a TOT state were related to the target word. Because the syllable is a more obvious component of a word than the first letter, we felt it was necessary to increase the number of unrelated words in each prime list to further disguise the nature of the primes. The unrelated list contained 10 filler words that were phonologically and semantically different from the target. The three "primed" lists consisted of three primes and seven filler words. Primes shared a single syllable with the target- the first, middle, or last syllable (see Appendix B); phonology was verified in Webster's New World College Dictionary (Neufeldt \& Guralnik, 1997). The two middle syllables of foursyllable words were primed in different words by having some primes with the first middle syllable and other primes that contained the second middle syllable. Filler words in all lists ranged from one to five syllables. The primes were positioned in the list so that no two primes appeared consecutively; one third of the word lists included primes at Positions 1, 4, and 8 in the list, one third of the word lists had primes in Positions 2, 5, and 9, and the last third of the word lists placed primes in Positions 3, 6, and 10.

Design and Procedure. The experiment featured a single-factor design with priming condition (first-syllable primes, middle-syllable primes, last-syllable primes, and unrelated fillers) as a withinparticipants factor. The procedure was the same as that in Experiment 1, with one exception: After responding "know" initially, participants received a subset of the unrelated list that contained either four or six words, instead of five as in Experiment 1.

\section{Results and Discussion}

The postexperimentquestionnaire revealed that $39 \%$ of participants reported no awareness of a relation between primes and targets, and 24\% reported awareness but incorrectly described a relation. In contrast, $37 \%$ of the participants correctly noticed a relation. When asked about intent to use the relation, 8 participants (13\%) reported intentionally and successfully using the primes to facilitate retrieval; that is, they reported that some prime words shared sounds with the target word and that they tried to 
use the primes to retrieve words at least once. These 8 participants were excluded from all subsequent analyses, as was 1 additional participant who did not complete the postexperiment questionnaire, resulting in 51 participants included in subsequent analyses.

In terms of the correct initial responses to the general knowledge questions, $27 \%$ were "know" responses, $16 \%$ were TOT responses, and $36 \%$ were "don't know" responses. As in Experiment 1, incorrect "know" responses (16\%) and incorrect TOT responses (5\%) were excluded from all analyses. The means and standard deviations for the percentage of resolution following each syllable priming condition are displayed for TOT responses in Table 1. Resolution following TOT responses was analyzed with a repeated measures analysis of variance (ANOVA). For TOT responses, 14 participants $(27.5 \%$ ) were excluded from analysis because of an insufficient number of TOT responses in every priming condition. The priming condition effect for TOT responses was significant $[F(3,108)=$ $\left.6.07, M S_{\mathrm{e}}=.10, p<.001\right]$. Post hoc tests revealed significantly greater resolution following first-syllable primes than following unrelated words $(p<.003)$. Resolution following middle-syllable primes $(p>.285)$ and lastsyllable primes $(p>679)$ did not significantly differ from resolution following unrelated words.

Even though participants who claimed to intentionally use the primes to resolve TOT states were excluded from all analyses, we were interested in whether these participants experienced more first-syllable priming than participants who did not use intentional retrieval strategies. Excluding the same 14 participants who did not have TOT states in all four priming conditions, a 2 (intent: successfully used the primes, no/unsuccessful intent) $\times 2$ (priming condition: first syllable, unrelated) ANOVA on resolution following TOT responses revealed only a significant priming condition main effect $\left[F(1,42)=7.07, M S_{\mathrm{e}}=.11, p<.011\right]$. Neither the main effect of intent nor the intent $\times$ priming condition interaction was significant $(F \mathrm{~s}<1)$. Only 7 participants were included in the successful intent group (one was excluded for not having TOTs in both priming conditions), making it difficult to achieve significance, but the priming effects of the two groups (the difference in resolution between first-syllable primes and unrelated words) were remarkably similar (priming effect for successful intent group $=26.6 \%$, priming effect for no/unsuccessful intent group $=25.5 \%$ ). Thus, it appears that participants who used a strategy to resolve their TOT states on occasion were no more likely to resolve TOT states following first-syllable prime words than were those who did not employ a strategy.

Unlike Experiment 1, this experiment demonstrated significant priming: Primes containing the first syllable of the target increased retrieval of the target. This priming effect is consistent with the TD model of TOTs, which postulates that phonological nodes receive insufficient priming for activation. When first syllables of target words were provided, these phonological nodes increased the transmission of priming to the remaining phonological nodes, causing an increase in TOT resolution. This priming effect cannot be explained by conscious search strategies to link the primes with the target word because participants reporting those strategies were excluded from analysis, and furthermore, these participants displayed amounts of priming equivalent to that of participants who reported an inability to use such strategies. The fact that only first-syllable primes facilitated TOT resolution relative to middleand last-syllable primes is consistent with prior research supporting the importance of initial phonology in aiding retrieval (Freedman \& Landauer, 1966; Gruneberg \& Monks, 1974; Meyer \& Bock, 1992; Perfect \& Hanley, 1992) and extends this finding to priming paradigms.

\section{EXPERIMENT 3}

The results of Experiments 1 and 2 suggest a difference in phonological priming of TOT resolution as a function of the phonology primed. However, this finding needs replication for several reasons. First, the lack of firstletter priming in Experiment 1 is counterintuitive to the TD model and therefore deserves replication as an unexpected finding. Second, differences in stimuli used in Experiments 1 and 2 could potentially contribute to the differences in priming between first-syllable and firstphoneme primes. Third, Experiment 2 included some targets whose first syllable consisted only of a single letter, confounding them with the first-letter condition. Fourth, the initial phonology between first-letter primes and targets in Experiment 1 did not always match, which could have resulted in less phonological priming. These issues were addressed in Experiment 3 by more carefully controlling the stimuli to overcome these possible influences. In addition, the comparison of first-phoneme and firstsyllable primes was made within participants to ensure that the same items and same participants were utilized in both priming conditions.

Experiment 3 also assessed the role that word production (aloud or silent) plays in priming of TOT resolution. Within node structure theory, both aloud and silent production utilize the same phonological nodes; aloud production additionally requires the use of nodes in the muscle movement system (e.g., MacKay, 1987). Therefore, the TD model predicts that recent activation of nodes, either through aloud or silent production, will strengthen their connections accordingly and increase the transmission of priming to the phonological nodes suffering from the transmission deficits (e.g., Burke et al., 1991). Prior research on priming of TOT resolution has either only required aloud production (James \& Burke, 2000) or used production as a within-participants variable (the present Experiments 1 and 2, which could not be analyzed because of excessive data loss). In Experiment 3, we manipulated production as a between-participantsvariable to assess whether silent production (i.e., reading silently) increases activation of the missing phonological information during a TOT state.

\section{Method}

Participants. Eighty-seven undergraduate native speakers of English (20 male, 67 female; mean age 18.6 years, $S D=0.9$ ) were re- 
cruited from the same sources as those in Experiments 1 and 2, although none of the participants had participated in Experiment 1 or 2 .

Materials. As in Experiment 2, we felt that using first-syllable primes required additional measures to disguise the nature of the primes and reduce participants' potential awareness of the primes' relation to the targets. In this experiment, we increased the number of unrelated filler words to eight (and reduced the number of primes to two). We also asked participants to make pronunciation judgments about the primes so that the primes' presentation seemed more plausible as a separate task and less linked to the general knowledge questions.

Eighty general knowledge questions were chosen from Experiments 1 and 2 (14 came solely from Experiment 1; 6 came solely from Experiment 2; 52 were used in both experiments; 7 came from James \& Burke, 2000; and 1 was generated for a word listed in Francis \& Kučera, 1982). The primed lists consisted of two primes and eight filler words. Primes shared either the first syllable or the first phoneme of the target, which was verified by Webster's New World College Dictionary (Neufeldt \& Guralnik, 1997). There was no other phonological overlap between the primes and the target, and the filler words were phonologically and semantically unrelated to the target or its primes (see Appendix C). Prime words in the firstsyllable condition shared first-syllable phonology with the target. The number of letters in the first syllable of the targets varied between two and five letters but were at least two letters long so as to avoid similarity to the first-phoneme condition. In terms of firstsyllable length, $50 \%$ of the targets contained two letters, $45 \%$ contained three letters, $3.8 \%$ contained four letters, and $1.3 \%$ contained five letters. For the majority of targets (71 out of 80), each of the two primes also shared the same number of syllables as the target.

The prime words in the first-phoneme condition shared their first phoneme with the targets (and shared the same first letter $93.7 \%$ of the time). However, targets and primes did not share the same first syllable, so that the first-phoneme and first-syllable conditions would not be confounded. In addition, targets and primes shared the same second letter only $1.3 \%$ of the time. The two first-phoneme primes for a specific target shared the same second letter with each other only $1.3 \%$ of the time as well.

The unrelated condition contained the same eight unrelated filler words as the first-phoneme and first-syllable conditions. Instead of primes, two additional unrelated words were chosen that matched the syllable length of the primes from the two primed lists. In all conditions, the eight unrelated filler words varied randomly by syllable length. With respect to position, half of the targets had primes in Positions 2 and 8 of the 10 words, whereas the other half of the targets had primes in Positions 3 and 9.

The recognition test and postexperiment questionnaire were identical to those used in Experiments 1 and 2, except for items added or deleted in Experiment 3. Participants also completed a vocabulary test consisting of 25 multiple-choice questions so we could examine whether level of general knowledge impacts the likelihood of phonological priming.

Design and Procedure. The experiment used a $3 \times 2$ mixed factorial design with one within-participants factor, priming condition (first-syllable primes, first-phoneme primes, and unrelated words), and one between-participants factor, production of primes (aloud vs. silent). The procedure was similar to that of Experiments 1 and 2 . After presentation of the general knowledge question, participants responded that they knew the answer, did not know, or were in a TOT state. After responding "know" initially, participants received a subset of the unrelated list that contained seven words. If they did not know the answer or were in a TOT state, a primed list (first syllable or first phoneme) or an unrelated list, each containing 10 words, was presented. Half of the participants read the words silently, and half read them aloud. After reading each word, participants verbally rated how easy or difficult a word was to pronounce on a scale of 1 (easy) to 7 (difficult). These words were presented one at a time and stayed on the screen until the participant made a pronounceability judgment. The general knowledge question was presented again for the participants to attempt word retrieval. If participants still did not know the word or were experiencing a TOT state, the participants were asked to give any "partial" information about the missing word. After all 80 general knowledge questions had been presented, a fourchoice recognition test was given for unresolved questions (i.e., "don't know" and TOT responses), followed by the postexperiment questionnaire.

\section{Results and Discussion}

Of the 87 participants tested, 3 participants $(3.5 \%$; 1 from the aloud condition and 2 from the silent condition) were excluded from analyses because they indicated awareness of the phonological relation between the primes and the targets and attempted to use this relation to facilitate their retrieval, as assessed by the postexperiment questionnaire. The majority of participants did not detect this relation $(79 \%)$ or detected a nonexistent relation $(14 \%)$, and the remaining participants $(3.5 \%)$ noticed the relation but declined using it to aid retrieval. The percentage of "aware" participants was considerably lower than that found in Experiment 2 (37\%), suggesting that methodological attempts to minimize detection of the relation between primes and targets were successful.

The percentages of initial responses to general knowledge questions were similar to those found in Experiments 1 and 2. In terms of categories, correct responses consisted of 30\% "know" responses, $13 \%$ TOT responses, and 38\% "don't know" responses. As in Experiments 1 and 2, incorrect "know" responses (14\%) and incorrect TOT responses $(5 \%)$ were excluded from all analyses. The means and standard deviations for the percentage of resolution following each priming condition are displayed for TOT responses in Table 2. Resolution following TOT responses was analyzed with a $3 \times 2$ repeated measures ANOVA, which excluded 5 participants $(6 \%)$ because they did not have at least one correct TOT response in each of the priming conditions. The ANOVA showed that there was a main effect of priming condition $\left[F(2,154)=6.09, M S_{\mathrm{e}}=\right.$ $0.08, p<.003]$, with a greater percentage of TOT states being resolved following first-syllable primes than following either first-phoneme primes $(p<.016)$ or unrelated words $(p<.002)$; there was no significant difference in TOT resolution between first-phoneme and unrelated conditions $(p>.426)$. Although the main effect of production of primes was not significant $(F<1)$, the priming condition $\times$ production of primes interaction was significant $\left[F(2,154)=3.52, M S_{\mathrm{e}}=0.08, p<.032\right]$. Post hoc tests showed that there were significant differences between priming conditions in silent production $\left[F(2,154)=9.14, M S_{\mathrm{e}}=0.08, p<.001\right]$, but not in aloud production $(F<1)$. For primes read silently, first-syllable primes yielded greater resolution than did either firstphoneme primes $(p<.009)$ or unrelated words $(p<$ $.001)$, whereas first-phoneme primes showed resolution equivalent to that of unrelated words $(p>.132)$. Analyses of this interaction within each priming condition revealed that first-syllable primes read silently led to mar- 
ginally greater TOT resolution than did first-syllable primes read aloud $\left[F(1,77)=3.75, M S_{\mathrm{e}}=0.11, p<\right.$ .057]. In contrast, there was no difference between aloud and silent production in TOT resolution for first-phoneme primes $(p>.755)$ or unrelated words $(p>.171)$.

To further explore the differences in first-syllable priming between aloud and silent production, a 2 (production of primes: aloud, silent) $\times 2$ (prime order: first prime, second prime) ANOVA was conducted on mean pronunciation ratings of the two primes in the first-syllable priming condition. This analysis yielded a main effect of production of primes $\left[F(1,77)=5.20, M S_{\mathrm{e}}=2.77, p<\right.$ .025]; primes read aloud had significantly higher (more difficult) pronunciation ratings $(M=2.99)$ than did primes read silently $(M=2.39)$. The main effect of prime order was not significant $\left[F(1,77)=1.02, M S_{\mathrm{e}}=.75, p>\right.$ $.314]$, nor was the production of primes $\times$ prime order interaction $\left[F(1,77)=1.95, M S_{\mathrm{e}}=.75, p>.111\right]$. We also examined the correlations between the probability of TOT resolution in the first-syllable condition and the pronunciation ratings of the two primes. For primes pronounced aloud, there was a significant negative correlation with each of the two primes' pronunciation ratings $[r(40)=$ $-.37, p<.02$, and $r(40)=-.30, p<.056$ ]; TOT resolution decreased as pronunciation rating increased (word was rated as harder to pronounce). In contrast, there were no significant correlations with either pronunciation rating for the two primes read silently $[r(39)=.00, p>.992$, and $r(39)=.08, p>.62$ ].

Correlational analyses were then conducted to determine whether vocabulary score was related to the percentage of TOT resolution following each priming condition. Vocabulary score ranged from 8 to 22 (out of 25 possible; $M=14.5, S D=3.2$ ). There was a significant correlation between vocabulary score and the percent of resolution following first-syllable primes $[r(79)=.32$, $p<.004]$. In contrast, there was no significant correlation between vocabulary score and TOT resolution following first-phoneme primes $[r(79)=.12, p>.282]$ or following unrelated primes $[r(79)=-.06, p>.577]$.

Experiment 3 replicated and extended the findings from Experiments 1 and 2 using a within-participants design, demonstrating that first-syllable primes facilitated word retrieval relative to unrelated words, whereas firstphoneme primes did not. The lack of phonological priming suggests that the first phoneme does not provide sufficient activation to the appropriate syllable to activate and

Table 2

Experiment 3: Word Retrieval (Percent)

Following Primes and Unrelated Words, Read Silently or Aloud, After an Initial TOT Response

Production of Prime

\begin{tabular}{lccccc}
\cline { 2 - 3 } & \multicolumn{2}{c}{ Words Read Silently } & & \multicolumn{2}{c}{ Words Read Aloud } \\
\cline { 2 - 3 } \cline { 5 - 6 } Priming Condition & Mean Resolution & $S D$ & & Mean Resolution & $S D$ \\
\hline Same first syllable & 50.2 & 33.9 & & 35.5 & 33.7 \\
Same first phoneme & 32.4 & 29.2 & & 30.1 & 35.8 \\
Unrelated & 22.9 & 29.6 & & 32.4 & 31.4 \\
\hline
\end{tabular}

therefore retrieve the entire word. In contrast, the first syllable provides sufficient phonological information to transmit priming to the remaining phonological nodes in order to activate them. The correlational analyses between vocabulary score and TOT resolution also support a fundamental difference between first-syllable and first-phoneme primes because vocabulary correlates with TOT resolution for first-syllable primes but not first-phoneme primes.

The most surprising finding of Experiment 3 was that reading first-syllable primes silently produced significant priming, whereas reading first-syllable primes aloud did not. Our explanation is that the addition of the pronunciation rating task encouraged participants to focus on their pronunciations when reading primes aloud, which may have adversely affected their TOT resolution. The experimenter noted that participants in the aloud condition often made pronunciation errors and showed concern about their performance when doing so. One possibility within the TD model is that mispronunciations allow the activation of inappropriate (nontarget) phonology, leading to a decrease in activation of the target's phonology. Given that mispronunciations are more likely to occur on words that are more difficult to pronounce, we would expect TOT resolution to decrease for these more difficult words. The analyses of the pronunciation ratings lend support to this argument: Primes read aloud were rated as more difficult to pronounce than primes read silently, and increased difficulty in pronunciation correlated negatively with TOT resolution. No such relationship was seen for primes read silently.

\section{GENERAL DISCUSSION}

The results of the three experiments presented here support a dissociation among phonological aspects of words in facilitating TOT resolution. Processing phonologically related words containing the first letter or phoneme did not increase resolution of TOT states, nor did primes containing the middle or last syllable of the target. In contrast, processing phonologically related words having the target's first syllable did increase correct recall of the target. This result suggests that the first syllable is a critical component for word retrieval and that the additional phonemes beyond the first phoneme are able to offset transmission deficits by activating phonological representations shared with the target, thereby strengthening connections and enabling target retrieval.

This first-syllable priming effect in Experiments 2 and 3 was predicted by node structure theory, which specifies a sequential nature of activation of phonology and therefore places importance on the initial part of a word for achieving activation of the entire word. It is worth noting that when significant first-syllable priming occurred, the effect sizes in Experiments 2 and 3 were similar (26\% in Experiment 2 and 27\% in Experiment 3), despite a difference in the number of primes presented-Experiment 2 used three primes, whereas Experiment 3 used two primes. This result suggests that the first syllable is a rather salient phonologicalcharacteristic that reliably aids TOT resolution independent of number of repetitions of this phonology. 
The difference between first-phoneme and first-syllable primes in influencing TOT resolution can be explained within the TD model, despite our initial predictions. Activation of the first phoneme sends node priming to a large neighborhood of first-syllable nodes that also contain that initial phoneme. Therefore, the target word's first syllable may not receive enough node priming to get activated because many other first-syllable nodes are receiving node priming from the first phoneme. In contrast, activation of the first syllable sends node priming to other lexical nodes (words) containing that first syllable, but there are considerably fewer words that share the same first syllable relative to syllables within words that share the same first phoneme. Thus, the likelihood of accumulating enough node priming on the target's lexical node to achieve activation is greater following first-syllable primes than firstphoneme primes.

The effects of first-syllable phonology on TOT resolution were not due to the utilization of conscious retrieval strategies. The findings from the postexperiment questionnaire support the implicit nature of our paradigmparticipants were most often unaware of a relation between the primes and the TOT target (i.e., awareness) and were generally unable to utilize the relation to strategically retrieve the target word even when aware of the relation (i.e., intent). This pattern was found in all three experiments despite differential priming effects occurring, further suggesting that knowledge of a relation between primes and targets cannot be the cause of increased TOT resolution following first-syllable primes. Furthermore, unlike typical repetition priming paradigms, intentional retrieval in the present experiments could not be used consistently because of the random assignment of the lists of prime words. The fact that participants claiming to use retrieval strategies had first-syllable priming equivalent to that of participants who denied using any explicit strategies suggests that intentional retrieval occurred infrequently and did not facilitate retrieval.

Experiment 3 revealed a positive correlation between vocabulary score and TOT resolution, which can also be explained by the TD model. People with better vocabularies are likely to utilize these low-frequency words more, which strengthens their lexical-to-phonological connections. Thus, even when these connections weaken and become susceptible to TOT states, the likelihood of reactivating this weakened phonology is greater for people with higher than lower vocabularies. The fact that this correlation exists only for first-syllable primes and not firstphoneme primes supports the claim that only first-syllable phonology is sufficient for activation of the entire word. Another possible explanation for this correlation is that people with weaker vocabularies may not have had true TOT states; that is, they incorrectly reported being in a TOT state when they really did not know the word. This trend is demonstrated in the present data by a negative correlation between vocabulary score and number of incorrect TOTs $[r(79)=-.30, p<.008]$. Therefore, priming low-vocabulary people with the target word's first syllable would not increase their resolution rates since the word's lexical node was not appropriately activated.

Another variable, production of primes, was also related to TOT resolution. Although the first-syllable priming effect was not demonstrated for primes read aloud in Experiment 3, we argue that this result was an artifact of the pronunciation rating task. The more important point is that across experiments, both types of production of the first syllable - aloud as well as silent—increased word retrieval relative to unrelated words. The only difference between aloud production and silent production in node structure theory is the usage of muscle movement nodes; both forms of production utilize identical phonological nodes. The present experiments demonstrate that TOT resolution can be increased when the necessary phonological components (i.e., first syllable) receive sufficient transmission of priming to enable activation, even when these components are activated via silent production.

By showing that reading silently can activate phonology, the present experiments also suggest that the pop-ups we experience during naturally occurring TOT states may result from other sources besides our own overt speech production-for example, through reading the essential phonology, or even from our own thoughts. Given that internal production and perception use the same nodes at all levels in node structure theory, will perception of primes, like production of primes, increase TOT resolution? This hypothesis could be tested in naturally occurring TOT states by assessing other people's production of essential phonological components and in laboratory TOT states through auditory presentation of primes. Similar to silent production, the extension of phonological priming effects to perception would provide additional evidence of the TD model's assumption of the underlying mechanism: a change in connection strength at the phonologicallevel, independent of nodes required for overt production of speech.

\section{REFERENCES}

ANDERson, J. R. (1983). A spreading activation theory of memory. Journal of Verbal Learning \& Verbal Behavior, 22, 261-295.

Bowers, J. S., \& SCHACTER, D. L. (1990). Implicit memory and test awareness. Journal of Experimental Psychology: Learning, Memory, \& Cognition, 16, 404-416.

Bowles, N. L., \& Poon, L. W. (1985). Effects of priming in word retrieval. Journal of Experimental Psychology: Learning, Memory, \& Cognition, 11, 272-283.

Brown, A. S. (1991). A review of the tip-of-the-tongue experience. Psychological Bulletin, 109, 204-223.

Brown, R, \& McNeill, D. (1966). The "tip of tongue" phenomenon. Journal of Verbal Learning \& Verbal Behavior, 5, 325-337.

Burke, D. M., MacKay, D. G., Worthley, J. S., \& Wade, E. (1991). On the tip of the tongue: What causes word finding failures in young and older adults? Journal of Memory \& Language, 30, 542-579.

Collins, A. M., \& LofTus, E. F. (1975). A spreading-activation theory of semantic processing. Psychological Review, 82, 407-428.

Francis, W. N., \& KuČERA, H. (1982). Frequency analysis of English Usage: Lexicon and Grammar. Boston: Houghton Mifflin.

Freedman, J. L., \& LANDAuer, T. K. (1966). Retrieval of long-term memory: "Tip-of-the-tongue" phenomenon. Psychonomic Science, 4, 309-310. 
Gruneberg, M. M., \& Monks, J. (1974). "Feeling of knowing" and cued recall. Acta Psychologica, 38, 257-265.

Harley, T. A., \& Bown, H. E. (1998). What causes a tip-of-the-tongue state? Evidence for lexical neighbourhood effects in speech production. British Journal of Psychology, 89, 151-174.

Heine, M. K., Ober, B. A., \& Shenaut, G. K. (1999). Naturally occurring and experimentally induced tip-of-the-tongue experiences in three adult age groups. Psychology \& Aging, 14, 445-457.

Horowitz, L. M., White, M. A., \& ATwood, D. W. (1968). Word fragments as aids to recall: The organization of a word. Journal of Experimental Psychology, 76, 219-226.

JAmes, L. E., \& Burke, D. M. (2000). Phonological priming effects on word retrieval and tip-of-the-tongue experiences in young and older adults. Journal of Experimental Psychology: Learning, Memory, \& Cognition, 26, 1378-1391.

Jones, G. V. (1989). Back to Woodworth: Role of interlopers in the tipof-the-tongue phenomenon. Memory \& Cognition, 17, 69-76.

Jones, G.V., \& LANGFORD, S. L. (1987). Phonologicalblocking in the tip of the tongue state. Cognition, 26, 115-122.

Kohn, S. E., Wingfield, A., Menn, L., Goodglass, H., Gleason, J. B., \& Hyde, M. (1987). Lexical retrieval: The tip-of-the-tongue phenomenon. Applied Psycholinguistics, 8, 245-266.

Koriat, A., \& Lieblich, I. (1975). Examination of the letter serial position effect in the "TOT" and the "don't know" states. Bulletin of the Psychonomic Society, 6, 539-541.

MACKAY, D. G. (1987). The organizationof perception and action: A theory for language and other cognitive skills. New York: Springer-Verlag.

MacKAY, D. G., \& Burke, D. M. (1990). Cognition and aging: A theory of new learning and the use of old connections. In T. Hess (Ed.),
Aging and cognition: Knowledge organization and utilization (pp. 213-263). Amsterdam: North-Holland.

MAYLOR, E. A. (1990). Age, blocking and the tip of the tongue state. British Journal of Psychology, 81, 123-134.

McKone, E. \& SleE, J. A. (1997). Explicit contamination in "implicit" memory for new associations. Memory \& Cognition, 25, 352-366.

MeYer, A. S., \& Bock, K. (1992). The tip-of-the-tongue phenomenon: Blocking or partial activation? Memory \& Cognition, 20, 715-726.

Miozzo, M., \& Caramazza, A. (1997). Retrieval of lexical-syntactic features in tip-of-the-tongue states. Journal of Experimental Psychology: Learning, Memory, \& Cognition, 23, 1410-1423.

Nelson, T. O., \& NARENS, L. (1980). Norms of 300 general-information questions: Accuracy of recall, latency of recall, and feeling-of-knowing ratings. Journal of Verbal Learning \& Verbal Behavior, 19, 338-368.

Neufeldt, V., \& GuRalniK, D. B. (Eds.) (1997). Webster's new world college dictionary (3rd ed.). New York: Simon \& Schuster.

Perfect, T. J., \& Hanley, J. R. (1992). The tip-of-the-tongue phenomenon: Do experimenter-presented interlopers have any effect? Cognition, 45, 55-75.

Rastle, K. G., \& Burke, D. M. (1996). Priming the tip of the tongue: Effects of prior processing on word retrieval in young and older adults. Journal of Memory \& Language, 35, 586-605.

Rubin, D. C. (1975). Within word structure in the tip-of-the-tonguephenomenon. Journal of Verbal Learning \& Verbal Behavior, 14, 392 397.

Yaniv, I., \& Meyer, D. E. (1987). Activation and metacognition of inaccessible stored information: Potential bases for incubation effects in problem solving. Journal of Experimental Psychology: Learning, Memory, \& Cognition, 13, 187-205.

\section{APPENDIX A \\ Example General Knowledge Question, Target Word, Primes, and Filler Words in Experiment 1}

Question: What do you call goods that are traded illegally (i.e., smuggled goods)?

Target Word: contraband

Primes in Experiment 1

\begin{tabular}{cc}
\hline First-Letter Primes & Unrelated Words \\
\hline crave & oriental \\
impervious & impervious \\
common & libertarians \\
bites & bites \\
antibodies & antibodies \\
calculation & spiked \\
nailed & nailed
\end{tabular}

$\overline{\text { Note-Prime words are underlined, and filler words are printed }}$ normally. 
APPENDIX B

Example General Knowledge Question, Target Word, Primes, and Filler Words in Experiment 2

Question: What do you call goods that are traded illegally (i.e., smuggled goods)? Target Word: contraband

Primes in Experiment 2

\begin{tabular}{llll}
\hline First Syllable & Middle Syllable & Last Syllable & Unrelated Words \\
\hline equator & equator & equator & equator \\
$\underline{\text { contact }}$ & demonstrable & hus $\underline{\text { band }}$ & appreciative \\
libertarians & libertarians & libertarians & libertarians \\
phonemics & phonemics & phonemics & phonemics \\
connotation & extradite & sweatband & persist \\
shriek & shriek & shriek & shriek \\
oriental & oriental & oriental & oriental \\
spiked & spiked & spiked & spiked \\
conscious & ultrasonic & headband & swimsuit \\
nailed & nailed & nailed & nailed \\
\hline
\end{tabular}

Note-Relevant portions of prime words are underlined, and filler words are printed normally.

\section{APPENDIX C \\ Example General Knowledge Question, Target Word, Primes, and Filler Words in Experiment 3}

Question: What do you call goods that are traded illegally (i.e., smuggled goods)?

Target Word: contraband

Primes in Experiment 3

\begin{tabular}{lll}
\hline First Phoneme & First Syllable & Unrelated Words \\
\hline thunder & thunder & thunder \\
rub & rub & rub \\
calico & constitute & presenting \\
oriental & oriental & oriental \\
libertarians & libertarians & libertarians \\
spiked & spiked & spiked \\
align & align & align \\
impervious & impervious & impervious \\
credential & congregate & brazenness \\
piston & piston & piston \\
\hline
\end{tabular}

Note-Relevant portions of prime words are underlined, and filler words are printed normally. 